\title{
Axisymmetric Longitudinal-Bending Waves in a Cylindrical Shell Interacting with a Nonlinear Elastic Medium
}

\author{
Alexander I. Zemlyanukhin, Andrey V. Bochkarev, \\ Lev I. Mogilevich, and Ekaterina G. Tindova \\ Department of Applied Mathematics and System Analysis, Yuri Gagarin State Technical University of Saratov, Saratov, Russia \\ Correspondence should be addressed to Andrey V. Bochkarev; ab2009sar@list.ru
}

Received 20 August 2016; Revised 26 October 2016; Accepted 1 November 2016

Academic Editor: Zhiping Qiu

Copyright (c) 2016 Alexander I. Zemlyanukhin et al. This is an open access article distributed under the Creative Commons Attribution License, which permits unrestricted use, distribution, and reproduction in any medium, provided the original work is properly cited.

\begin{abstract}
A nonlinear differential equation is derived which describes the propagation of axisymmetric stationary longitudinal-bending waves in infinite cylindrical shell of Timoshenko type, interacting with the external nonlinear elastic medium. A modified perturbation method based on the use of diagonal Pade approximants was applied to build exact solitary-wave solutions of the derived equation in the form of traveling front and the traveling pulse. Numerical solutions of the equation, obtained by means of finite difference method, are in good agreement with the corresponding exact analytical ones.
\end{abstract}

\section{Introduction}

The shell theory is a well-developed section of deformable solids mechanics and has numerous technical applications. The interest in nonclassical variants of shell theory has grown recently due to the need of analysing nanoobjects elastic properties, carbon nanotubes (CNTs), in particular [1-4].

Nonlinearity and dispersion are the main determinants of wave processes in elastic thin-walled structures. Longitudinal waves in rectilinear rods and plates interact with bending waves only in nonlinear approximation. Thus, longitudinal waves parametrically affect bending waves, which serve, in turn, as a nonlinear source for longitudinal waves. As for the shells the longitudinal and normal components of displacement are already connected in the linear approximation [5]. For this reason, the dynamic processes in shells are significantly more complex than similar processes in rods and plates.

Most of the tasks of wave propagation in elastic shells are considered in the linear formulation [6]. Analytical study of coupled equations system of shell element motion, written in displacements, is task of some difficulty; therefore researchers need to simplify it. Rather often, the longitudinal displacements' inertia is neglected; that is, the shell middle surface is considered to be unstretchable. For the axisymmetric case this allows us to integrate the first equation of the system and reduce the problem to solving a single equation for the normal displacement. This approach is traditional for onedimensional deformable systems and is often referred to as the Kirchhoff hypothesis [7]. However, the wave process in the shells cannot be adequately described with the use of such simplifications.

Equations of shell theory contain several small dimensionless parameters, the ratio of the thickness to the radius of curvature, in particular. Therefore, approximate solutions to these equations can naturally be obtained by means of the perturbation method [8], based on the small-parameter expansion of an unknown solution. The perturbation method is widely used to solve various problems of statics and dynamics of deformable structures. For example, in [9], the authors investigate the influence of small first-order and second-order perturbations of structural parameters such as stiffness and mass on the perturbations of structural vibration eigenvalues and eigenvectors. Traditionally, only the first few terms of the perturbation series are retained to find the approximate solution of the problem. However, the requirement of geometricity for this series allows us to find the sum of the series, which gives the problem exact solution [10]. 
The article aims at deriving and analysing the equation modeling the propagation of axisymmetric longitudinalbending waves in infinite cylindrical shell, interacting with a external nonlinear elastic medium $[11,12]$. The assumption of the infinity of the shell is valid if its edges are damped in such an optimal way that incident waves are not reflected [13]. Then, the influence of the boundary conditions can be ignored and vibrations propagation through the shell can be considered as traveling elastic waves. To find exact solutions to the derived equation we use the perturbation method with the Pade approximants [14]. In this paper we will restrict our search to solitary-wave (soliton-like) solutions.

\section{The Equation Derivation}

We start from the model of Timoshenko-type shell [15], which, in contrast to the Kirchhoff-Love model, takes into account the additional deformations associated with transverse forces and rotational inertia.

The equations of motion of cylindrical shell element under the assumption of independence of the wave process from the circumferential coordinate have the form

$$
\begin{aligned}
& \frac{\partial^{2} u}{\partial x^{2}}-\mu k_{y} \frac{\partial w}{\partial x}-\frac{\gamma}{g} \frac{1-\mu^{2}}{E} \frac{\partial^{2} u}{\partial t^{2}}=0, \\
& k^{2} \frac{1-\mu}{2}\left(\frac{\partial^{2} w}{\partial x^{2}}+\frac{\partial \psi}{\partial x}\right)+\mu k_{y} \frac{\partial u}{\partial x}-k_{y}^{2} w \\
& \quad-\frac{\gamma}{g} \frac{1-\mu^{2}}{E} \frac{\partial^{2} w}{\partial t^{2}}=k_{1} w+k_{2} w^{3} \\
& \frac{\partial^{2} \psi}{\partial x^{2}}-\frac{\gamma}{g} \frac{1-\mu^{2}}{E} \frac{\partial^{2} \psi}{\partial t^{2}}-6 k^{2} \frac{1-\mu}{h^{2}}\left(\frac{\partial w}{\partial x}+\psi\right)=0,
\end{aligned}
$$

where $x, z$ are longitudinal and radial coordinates; $u, w$ are displacements of points of the shell's middle surface in the directions $x, z ; \psi$ is the angle of rotation of a normal to the middle surface, $E$ is Young's modulus, $\mu$ is Poisson's ratio, $k_{y}=$ $1 / R$ is the parameter of curvature, $R$ is the radius of curvature of the shell, $\gamma$ is the specific weight of the shell material, $g$ is the gravitational acceleration, $t$ is time, $h$ is the shell thickness, and $k$ is the correction factor of the Timoshenko model; and $k_{1}, k_{2}$ are the coefficients characterizing the influence of external nonlinear elastic medium (Figures 1 and 2).

Solutions of system (1) will be sought in the class of stationary waves:

$$
\begin{aligned}
& u=u(\xi), \\
& w=w(\xi), \\
& \psi=\psi(\xi),
\end{aligned}
$$

where $\xi=x-V t$ is "running coordinate" and $V$ is the constant wave speed. Equations (1) take the form

$$
\left(1-k_{4}\right) \frac{d^{2} u}{d \xi^{2}}-\mu k_{y} \frac{d w}{d \xi}=0
$$

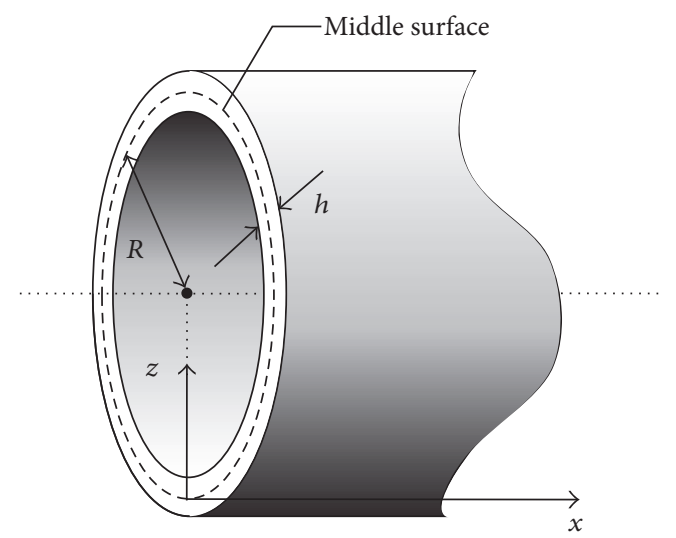

FIgURE 1: Geometry of cylindrical shell.

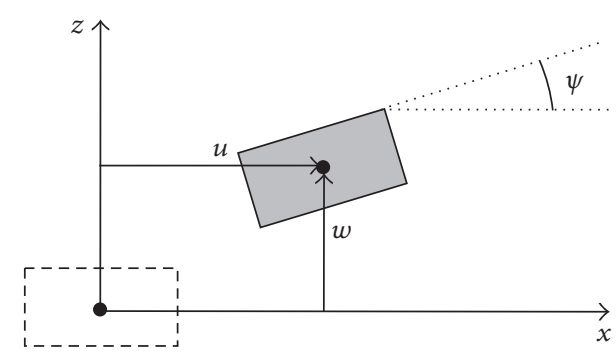

FIGURE 2: Displacements and rotation of shell element.

$$
\begin{aligned}
& k_{3}\left(\frac{d^{2} w}{d \xi^{2}}+\frac{d \psi}{d \xi}\right)+\mu k_{y} \frac{d u}{d \xi}-k_{y}^{2} w-k_{4} \frac{d^{2} w}{d \xi^{2}} \\
& =k_{1} w+k_{2} w^{3} \\
& \left(1-k_{4}\right) \frac{d^{2} \psi}{d \xi^{2}}-6 k^{2} \frac{1-\mu}{h^{2}}\left(\frac{d w}{d \xi}+\psi\right)=0,
\end{aligned}
$$

where $k_{3}=k^{2}((1-\mu) / 2)$ and $k_{4}=V^{2}(\gamma / g)\left(\left(1-\mu^{2}\right) / E\right)$.

The relationship between normal and longitudinal displacements is determined from (3):

$$
\frac{d u}{d \xi}=\frac{\mu k_{y}}{1-k_{4}} w
$$

By substituting (6) into (4), we express the derivative of the normal's rotation angle $d \psi / d \xi$ through the component of normal displacement $w$ :

$$
\begin{aligned}
\frac{d \psi}{d \xi} & =-\frac{1}{k_{3}}\left[\left(k_{3}-k_{4}\right) \frac{d^{2} w}{d \xi^{2}}+\left(\frac{\mu^{2} k_{y}^{2}}{1-k_{4}}-k_{y}^{2}-k_{1}\right) w\right. \\
\left.-k_{2} w^{3}\right] . &
\end{aligned}
$$

By differentiating equation (5) by $\xi$ and substituting the derivative (7) into the resulting expression, we finally get a 
nonlinear ordinary differential equation for the component of normal displacement

$$
\frac{d^{4} w}{d \xi^{4}}+a_{1} \frac{d^{2} w}{d \xi^{2}}+a_{2} \frac{d^{2}\left(w^{3}\right)}{d \xi^{2}}+a_{3} w+a_{4} w^{3}=0
$$

where

$$
\begin{aligned}
& a_{1}=-\frac{1}{k_{3}-k_{4}}\left(-\frac{h^{2} \mu^{2} k_{y}^{2}+12 k_{3} k_{4}}{h^{2}\left(1-k_{4}\right)}+k_{y}^{2}+k_{1}\right), \\
& a_{2}=-\frac{k_{2}}{k_{3}-k_{4}}, \\
& a_{3}=\frac{12 k_{3}}{\left(1-k_{4}\right)\left(k_{3}-k_{4}\right) h^{2}}\left(-\frac{\mu^{2} k_{y}^{2}}{1-k_{4}}+k_{y}^{2}+k_{1}\right), \\
& a_{4}=\frac{12 k_{2} k_{3}}{\left(1-k_{4}\right)\left(k_{3}-k_{4}\right) h^{2}} .
\end{aligned}
$$

Note that the equation similar to (8) was previously obtained in [16] for bending waves in Timoshenko-type beam lying on a nonlinear elastic foundation. The qualitative analysis of this equation was carried out for the case $a_{3}=a_{4}=$ 0 . The full equation is considered in this paper.

\section{Solitary-Wave Solutions}

To find exact solitary-wave solutions of equation (8) we will use the direct perturbation method with the Pade approximants [14], proposed in [10]. The basic idea of the method consists in establishing the relationships between the equation coefficients and parameters of the sought solution in which the perturbation series becomes geometric. In this case the sum of the series gives the expression for the sought solution. The criterion of geometricity of the series is the equality of sequential diagonal Pade approximants [Q/Q], $[Q+1 / Q+$ $1],[Q+2 / Q+2], \ldots$, the minimum order $Q$ of which is determined by the pole order of the sought solution.

The pole order of the solution is determined from the balance of the dominant terms of equation [17]. By substituting the function $w=a z^{-p}$, where $a$ and $p$ are constant, in the left part of (8), we obtain the expression

$$
\begin{aligned}
& a p(p+1)(p+2)(p+3) z^{-p-4} \\
& \quad+3 a_{2} a^{3} p(3 p+1) z^{-3 p-2}+a_{1} a p(p+1) z^{-p-2} \\
& \quad+a_{3} a z^{-p}+a_{4} a^{3} z^{-3 p}
\end{aligned}
$$

the first two terms of which are dominant. The balance is achieved by the equality of degrees $(-p-4)=(-3 p-2)$ from which we obtain $p=1$. Thus, the solution has a pole of the 1st order and it is necessary to choose $Q=1$ for the Pade approximants.

According to the perturbation method [8], we seek a solution of equation (8) in the form of functional series

$$
w=\sum_{n=1}^{\infty} \varepsilon^{n} w_{n}(\xi)
$$

where $w_{n}(\xi)$ are functions to be determined and $\varepsilon$ is a formal parameter. By substituting (11) into (8) and grouping in powers of $\varepsilon$, we obtain the infinite system of equations for the functions $w_{n}(\xi)$ :

$$
\begin{aligned}
\varepsilon^{1}: & \frac{d^{4} w_{1}}{d \xi^{4}}+a_{1} \frac{d^{2} w_{1}}{d \xi^{2}}+a_{3} w_{1}=0, \\
\varepsilon^{2}: & \frac{d^{4} w_{2}}{d \xi^{4}}+a_{1} \frac{d^{2} w_{2}}{d \xi^{2}}+a_{3} w_{2}=0, \\
\varepsilon^{3}: & \frac{d^{4} w_{3}}{d \xi^{4}}+a_{1} \frac{d^{2} w_{3}}{d \xi^{2}}+a_{3} w_{3}=-a_{4} w_{1}^{3} \\
& -3 a_{2} w_{1}\left[w_{1} \frac{d^{2} w_{1}}{d \xi^{2}}+2\left(\frac{d w_{1}}{d \xi}\right)^{2}\right], \\
\varepsilon^{4}: & \frac{d^{4} w_{4}}{d \xi^{4}}+a_{1} \frac{d^{2} w_{4}}{d \xi^{2}}+a_{3} w_{4}=-3 a_{4} w_{1}^{2} w_{2} \\
& -3 a_{2}\left(2 w_{1} w_{2} \frac{d^{2} w_{1}}{d \xi^{2}}+w_{1}^{2} \frac{d^{2} w_{2}}{d \xi^{2}}+4 w_{1} \frac{d w_{1}}{d \xi} \frac{d w_{2}}{d \xi}\right. \\
& \left.+2 w_{2}\left(\frac{d w_{1}}{d \xi}\right)^{2}\right)
\end{aligned}
$$

The first equation of system (12) has particular solution $w_{1}=$ $e^{\xi}$ under the condition of

$$
a_{1}=-a_{3}-1
$$

The equality $w_{2}=0$ must be chosen as a solution to the second equation of system (12). This allows us to obtain the solution of the $n$th equation of the system in the form of $w_{n}=K_{n} w_{1}^{n}$. By sequentially solving the other equations and determining the constants $K_{n}$ for decomposition (11), we have

$$
\begin{aligned}
w= & \varepsilon e^{\xi}+\frac{\left(9 a_{2}+a_{4}\right)}{8\left(a_{3}-9\right)}\left(\varepsilon e^{\xi}\right)^{3}+\frac{\left(9 a_{2}+a_{4}\right)\left(25 a_{2}+a_{4}\right)}{64\left(a_{3}-9\right)\left(a_{3}-25\right)}\left(\varepsilon e^{\xi}\right)^{5} \\
& +\frac{\left(9 a_{2}+a_{4}\right)\left(49 a_{2}+a_{4}\right)\left(17 a_{2} a_{3}+a_{3} a_{4}-225 a_{2}-17 a_{4}\right)}{512\left(a_{3}-9\right)^{2}\left(a_{3}-25\right)\left(a_{3}-49\right)}\left(\varepsilon e^{\xi}\right)^{7}+\cdots
\end{aligned}
$$


The right part of equality (14) contains only odd degree of product $\varepsilon e^{\xi}$. By multiplying both parts of the equality by $\varepsilon e^{\xi}$ and denoting $z=\left(\varepsilon e^{\xi}\right)^{2}$, we obtain a power series containing all powers of the variable $z$ :

$$
\begin{aligned}
\widetilde{w} & =z+\frac{9 a_{2}+a_{4}}{8\left(a_{3}-9\right)} z^{2}+\frac{\left(9 a_{2}+a_{4}\right)\left(25 a_{2}+a_{4}\right)}{64\left(a_{3}-9\right)\left(a_{3}-25\right)} z^{3} \\
& +\frac{\left(9 a_{2}+a_{4}\right)\left(49 a_{2}+a_{4}\right)\left(17 a_{2} a_{3}+a_{3} a_{4}-225 a_{2}-17 a_{4}\right)}{512\left(a_{3}-9\right)^{2}\left(a_{3}-25\right)\left(a_{3}-49\right)} \\
& \cdot z^{4}+\cdots
\end{aligned}
$$

By calculating the diagonal Pade approximants [1/1] and [2/2] for series (15), we perform the factorization of their difference. The numerator of the resulting expression contains the multipliers

$$
\left(a_{3}-49\right)\left(9 a_{2}+a_{4}\right)\left(a_{2} a_{3}+a_{4}\right) .
$$

The first multiplier (16) is in the denominators of (15) and cannot go to zero. The second multiplier is included in the numerators of all terms (15), except the first, and leads to a trivial solution $\widetilde{w}=z$. Finally, the third multiplier is reset to zero under the condition of

$$
a_{3}=-\frac{a_{4}}{a_{2}}
$$

which transforms the series (15) in the geometric one

$$
\widetilde{w}=z-\frac{a_{2}}{8} z^{2}+\frac{a_{2}^{2}}{64} z^{3}-\frac{a_{2}^{3}}{512} z^{4}+\cdots
$$

The sum of the series (18) coincides with approximants [1/1], $[2 / 2]$ and after returning to the variables $\xi$ and $w$, we have an equation

$$
\begin{aligned}
w & =\frac{8 \varepsilon e^{\xi}}{a_{2} \varepsilon^{2}\left(e^{\xi}\right)^{2}+8} \\
& = \pm \sqrt{\frac{2}{a_{2}}} \operatorname{sech}\left[\xi+\ln \left( \pm \varepsilon \sqrt{\frac{a_{2}}{8}}\right)\right]
\end{aligned}
$$

in which the signs are chosen the same as the sign of $\varepsilon$. The expression (19) contains two arbitrary parameters $\varepsilon, a_{2}$ and is an exact solution of equation (8) whose coefficients are connected by the relations (13) and (17). Under the assumption that $a_{2}>0$, the solution has the form of a traveling pulse. Note, that unlike the classical perturbation method, where $\varepsilon$ is a small parameter, in this case, $\varepsilon$ is an arbitrary real number.

Strictly speaking, a power series would be geometric or becomes so after regrouping the terms, if all diagonal Pade approximants $[Q / Q],[Q+1 / Q+1],[Q+2 / Q+2], \ldots$ coincide with each other. Condition (17) guarantees equality of only the first two approximants of the specified sequence. However, the substitution in (8) shows that (19) is the exact solution. Otherwise, we would require the equality of the two following approximants $[Q+1 / Q+1]$ and $[Q+2 / Q+2]$ to obtain the second equation for the coefficients entering the decomposition (15) and to check whether these approximants give exact solution. Such steps are repeated until an exact solution is found, either the system of equations for the coefficients will be inconsistent. In the latter case, the solution by this method is impossible to obtain.

To find a kink-type solution we need to substitute the expression

$$
w=E+\sum_{n=1}^{\infty} \varepsilon^{n} w_{n}(\xi)
$$

where $E$ is an arbitrary constant, into equation (8). After grouping by powers of $\varepsilon$ we have

$$
\begin{aligned}
\varepsilon^{0}: & E^{3} a_{4}+E a_{3}=0, \\
\varepsilon^{1}: & \frac{d^{4} w_{1}}{d \xi^{4}}+\left(3 E^{2} a_{2}+a_{1}\right) \frac{d^{2} w_{1}}{d \xi^{2}} \\
& +\left(3 E^{2} a_{4}+a_{3}\right) w_{1}=0 \\
\varepsilon^{2}: & \frac{d^{4} w_{2}}{d \xi^{4}}+\left(3 E^{2} a_{2}+a_{1}\right) \frac{d^{2} w_{2}}{d \xi^{2}} \\
& +\left(3 E^{2} a_{4}+a_{3}\right) w_{2}=-6 E a_{2} \frac{d}{d \xi}\left(w_{1} \frac{d w_{1}}{d \xi}\right) \\
& -3 E a_{4} w_{1}^{2}, \\
\varepsilon^{3}: & \frac{d^{4} w_{3}}{d \xi^{4}}+\left(3 E^{2} a_{2}+a_{1}\right) \frac{d^{2} w_{3}}{d \xi^{2}} \\
& +\left(3 E^{2} a_{4}+a_{3}\right) w_{3}=-6 E a_{2} \frac{d^{2}}{d \xi^{2}}\left(w_{1} w_{2}\right) \\
& -3 a_{2} \frac{d}{d z}\left(w_{1}^{2} \frac{d w_{1}}{d z}\right)-a_{4} w_{1}\left(6 E w_{2}+w_{1}^{2}\right)
\end{aligned}
$$

From the first equation of system (21) we find

$$
a_{4}=-\frac{a_{3}}{E^{2}}
$$

the second equation (21) has a particular solution $w_{1}=e^{\xi}$ if

$$
a_{1}=-3 E^{2} a_{2}+2 a_{3}-1
$$

the remaining equations (21) have the solutions in the form of $w_{n}=K_{n} w_{1}^{n}$. By sequentially determining constants $K_{n}, n=$ $2,3, \ldots$, we can represent the series from the right part of equality (20) in the form of 


$$
\begin{aligned}
\sum_{n=1}^{\infty} \varepsilon^{n} w_{n}= & z-\frac{4 E^{2} a_{2}-a_{3}}{2 E\left(a_{3}+2\right)} z^{2}+\frac{\left(6 E^{2} a_{2}-2 a_{3}-1\right)\left(9 E^{2} a_{2}-a_{3}\right)}{4 E^{2}\left(a_{3}+2\right)\left(2 a_{3}+9\right)} z^{3} \\
& -\frac{\left(28 E^{4} a_{2}^{2} a_{3}+72 E^{4} a_{2}^{2}-16 E^{2} a_{2} a_{3}^{2}-58 E^{2} a_{2} a_{3}-36 E^{2} a_{2}+2 a_{3}^{3}+9 a_{3}^{2}+8 a_{3}\right)\left(16 E^{2} a_{2}-a_{3}\right)}{8 E^{3}\left(a_{3}+2\right)^{2}\left(a_{3}+8\right)\left(2 a_{3}+9\right)} z^{3}+\cdots
\end{aligned}
$$

where $z=\varepsilon e^{\xi}$.

The numerator of the difference of the diagonal Pade approximants [1/1] and [2/2] for the series (24) contains the multipliers

$$
\begin{aligned}
& \left(a_{3}+8\right)\left(11 E^{2} a_{2} a_{3}-18 E^{2} a_{2}-4 a_{3}^{2}+2 a_{3}\right) \\
& \cdot\left(2 E^{2} a_{2}+1\right)
\end{aligned}
$$

the first of which cannot be zeroed, the second does not lead to exact solutions, and the third gives the condition

$$
a_{2}=-\frac{1}{2 E^{2}}
$$

under which the series (24) becomes geometric

$$
\sum_{n=1}^{\infty} \varepsilon^{n} w_{n}=z+\frac{z^{2}}{2 E}+\frac{z^{3}}{4 E^{2}}+\frac{z^{4}}{8 E^{3}}+\cdots
$$

and has the sum

$$
\frac{2 E z}{2 E-z}
$$

After substituting (28) instead of the series into (20) and returning to the variable $\xi$ we obtain the expression, being the exact solution of equation (8) under conditions (22), (23), and (26):

$$
w=E+\frac{2 E \varepsilon e^{\xi}}{2 E-\varepsilon e^{\xi}}=-E \tanh \left[\frac{1}{2}\left(\xi+\ln \left(-\frac{\varepsilon}{2 E}\right)\right)\right]
$$

The solution (29), containing two arbitrary constants $E$ and $\varepsilon$, is bounded and has the form of a traveling front (kink) if $E \varepsilon<0$.

\section{Numerical Results}

In this chapter, the verification is performed to ensure that the proposed analytical method is correct.

We use finite difference method [18] to obtain numerical solutions of the nonlinear ordinary differential equation (8). Difference scheme for solving the boundary value problem can be represented by using central differences:

$$
\begin{aligned}
& \frac{w_{j+2}-4 w_{j+1}+6 w_{j}-4 w_{j-1}+w_{j-2}}{h^{4}} \\
& +a_{1} \frac{w_{j+1}-2 w_{j}+w_{j-1}}{h^{2}} \\
& +a_{2} \frac{\left(w_{j+1}\right)^{3}-2\left(w_{j}\right)^{3}+\left(w_{j-1}\right)^{3}}{h^{2}}+a_{3} w_{j} \\
& +a_{4}\left(w_{j}\right)^{3}=0 .
\end{aligned}
$$

To linearize the difference equation (30), we use the following approximate replacement for the terms with cubic nonlinearity:

$$
\begin{aligned}
\left(w^{k+1}\right)^{3}= & \left(w^{k+1}\right)^{3}-\left(w^{k}\right)^{3}+\left(w^{k}\right)^{3} \\
= & \left(w^{k+1}-w^{k}\right) \\
& \cdot\left[\left(w^{k+1}\right)^{2}+w^{k+1} w^{k}+\left(w^{k}\right)^{2}\right]+\left(w^{k}\right)^{3} \\
\approx & \left(w^{k+1}-w^{k}\right) \cdot 3\left(w^{k}\right)^{2}+\left(w^{k}\right)^{3} \\
= & 3 w^{k+1}\left(w^{k}\right)^{2}-2\left(w^{k}\right)^{3},
\end{aligned}
$$

where $k$ denotes the iteration number. Substituting (31) into (30), we get the difference problem to be linear in the grid variable $\left\{w^{k+1}\right\}$

$$
\begin{aligned}
& w_{0}^{k+1}=\widetilde{w}\left(-\xi_{0}\right) \\
& w_{1}^{k+1}=\widetilde{w}\left(-\xi_{0}+h\right), \\
& \frac{w_{j+2}^{k+1}-4 w_{j+1}^{k+1}+6 w_{j}^{k+1}-4 w_{j-1}^{k+1}+w_{j-2}^{k+1}}{h^{4}}+a_{1} \frac{w_{j+1}^{k+1}-2 w_{j}^{k+1}+w_{j-1}^{k+1}}{h^{2}} \\
& \quad+a_{2} \frac{\left[3 w_{j+1}^{k+1}\left(w_{j+1}^{k}\right)^{2}-2\left(w_{j+1}^{k}\right)^{3}\right]-2\left[3 w_{j}^{k+1}\left(w_{j}^{k}\right)^{2}-2\left(w_{j}^{k}\right)^{3}\right]+\left[3 w_{j-1}^{k+1}\left(w_{j-1}^{k}\right)^{2}-2\left(w_{j-1}^{k}\right)^{3}\right]}{h^{2}}+a_{3} w_{j}^{k+1} \\
& \quad+a_{4}\left[3 w_{j}^{k+1}\left(w_{j}^{k}\right)^{2}-2\left(w_{j}^{k}\right)^{3}\right]=0, \quad j=\frac{2, N-3}{2,}
\end{aligned}
$$


TABLE 1: Deviations of the numerical solution $w_{n}$ from the exact solution $\widetilde{w}\left(\xi_{n}\right)(19)$.

\begin{tabular}{|c|c|c|c|c|c|}
\hline$h$ & $1.00 e-01$ & $5.00 e-02$ & $2.50 e-02$ & $1.25 e-02$ & $6.25 e-03$ \\
\hline \multicolumn{6}{|c|}{$a_{1}=1.0, a_{2}=1.0, a_{3}=-2.0, a_{4}=2.0, \varepsilon=2.0$} \\
\hline$\left\|w_{n}-\widetilde{w}\left(\xi_{n}\right)\right\|_{2}$ & $5.83 e-03$ & $2.05 e-03$ & $7.24 e-04$ & $2.54 e-04$ & $8.95 e-04$ \\
\hline \multicolumn{6}{|c|}{$a_{1}=0.0, a_{2}=1.0, a_{3}=-1.0, a_{4}=1.0, \varepsilon=2.0$} \\
\hline$\left\|w_{n}-\widetilde{w}\left(\xi_{n}\right)\right\|_{2}$ & $5.89 e-03$ & $2.07 e-03$ & $7.30 e-04$ & $2.71 e-04$ & $4.27 e-04$ \\
\hline$\left\|w_{n}-\widetilde{w}\left(\xi_{n}\right)\right\|_{2}$ & $4.16 e-03$ & $\begin{array}{r}.5, a_{2}=2.0 \\
1.46 e-03\end{array}$ & $\begin{array}{c}a_{4}=2.0, \varepsilon= \\
5.15 e-04\end{array}$ & $1.90 e-04$ & $4.90 e-04$ \\
\hline
\end{tabular}

TABLE 2: Deviations of the numerical solution $w_{n}$ from the exact solution $\widetilde{w}\left(\xi_{n}\right)(29)$.

\begin{tabular}{lcccr}
\hline$h$ & $1.00 e-01$ & $5.00 e-02$ & $2.50 e-02$ & $1.25 e-02$ \\
\hline$\left\|w_{n}-\widetilde{w}\left(\xi_{n}\right)\right\|_{2}$ & $9.19 e-03$ & $a_{1}=4.5, a_{2}=0.5, a_{3}=2.0, a_{4}=-2.0, E=1.0, \varepsilon=-1.0$ & $6.25 e-03$ \\
\hline$w_{n}-\widetilde{w}\left(\xi_{n}\right) \|_{2}$ & $5.14 e-03$ & $3.23 e-03$ & $1.14 e-03$ & $3.96 e-04$ \\
& $a_{1}=4.5, a_{2}=-0.5, a_{3}=2.0, a_{4}=-2.0, E=1.0, \varepsilon=-0.5$ & \\
$\left\|w_{n}-\widetilde{w}\left(\xi_{n}\right)\right\|_{2}$ & $1.36 e-02$ & $1.86 e-03$ & $6.69 e-04$ & $2.18 e-04$ \\
\hline
\end{tabular}

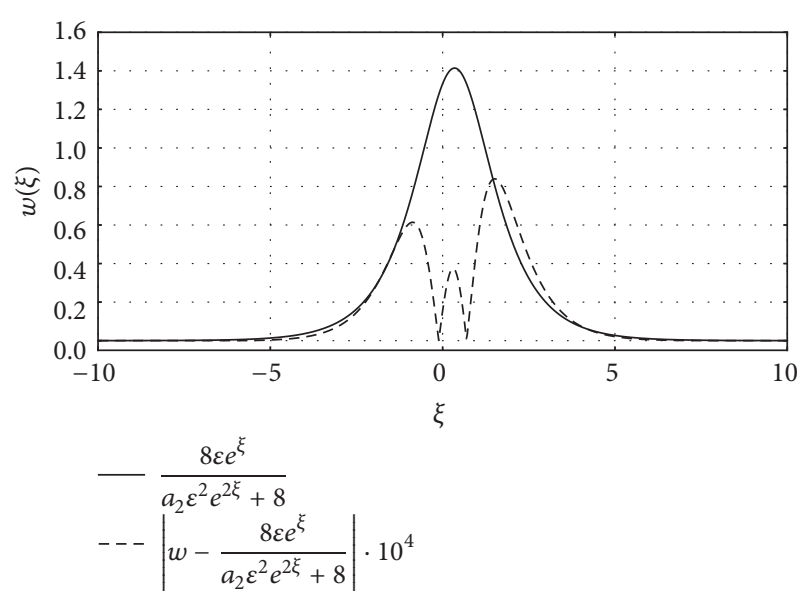

(a) Solution (19) of equation (8) at $a_{1}=1.0, a_{2}=1.0, a_{3}=-2.0, a_{4}=$ 2.0, and $\varepsilon=2.0$

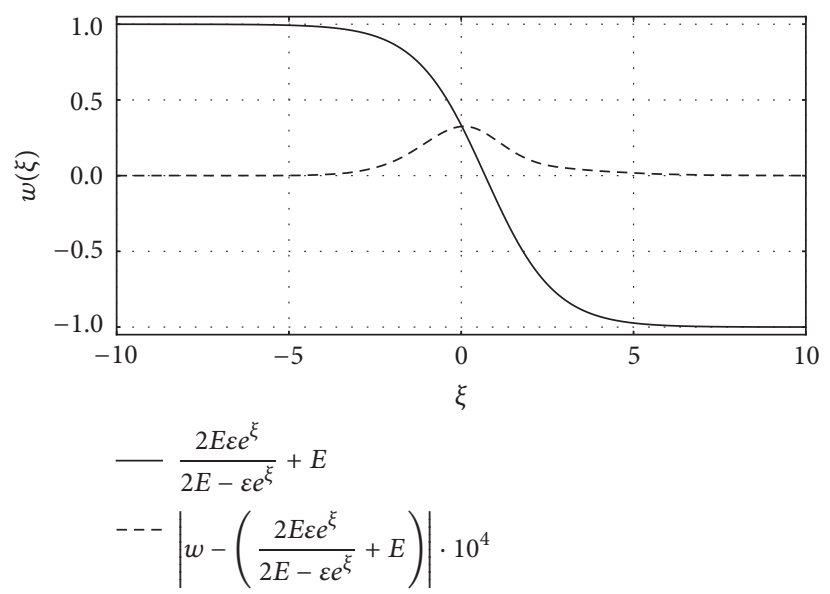

(b) Solution (29) of equation (8) at $a_{1}=4.5, a_{2}=0.5, a_{3}=2.0, a_{4}=$ $-2.0, E=1.0$, and $\varepsilon=-1.0$

FIGURE 3: The exact solution (solid line) and error of the numerical solution multiplied by $10^{4}$ (dashed line).

$$
\begin{aligned}
w_{N-2}^{k+1} & =\widetilde{w}\left(\xi_{0}-h\right), \\
w_{N-1}^{k+1} & =\widetilde{w}\left(\xi_{0}\right),
\end{aligned}
$$

where $\left\{w_{n}\right\}$ is a grid variable set on a uniform grid $\left\{\xi_{n}\right\}, \xi_{n}=$ $-\xi_{0}+n h, n=\overline{0, N-1} ; N=1+2 \xi_{0} / h$ is a number of grid nodes; $\left[-\xi_{0}, \xi_{0}\right]$ is an integration interval; $h$ is a grid spacing; $k$ and $(k+1)$ are the numbers of the previous and current iterations, respectively; and $\widetilde{w}(\xi)$ is an exact solution of equation (8).

The problem (32) is solved by means of fixed-point iteration method. The calculation process is stopped when $L^{2}$ norm of the difference between the solution from the current iteration and the solution from previous iteration is less than a specified small number $\alpha$ :

$$
\left\|w^{k+1}-w^{k}\right\|_{2} \equiv\left[\sum_{n=0}^{N-1}\left(w_{n}^{k+1}-w_{n}^{k}\right)^{2}\right]^{1 / 2}<\alpha .
$$

Deviations of the numerical solution from the exact solution for different values of the coefficients of equation (8) are shown in Tables 1 and 2. The calculations are performed under the conditions $\xi_{0}=10, \alpha=1.0 e-6$.

As shown in Figure 3, the curves of the exact solution and the numerical solution practically coincide: the maximum value of the absolute difference of the solutions does not exceed $0.83 e-4$.

\section{Conclusion}

The analysis showed that the search for solutions to the equations of motion of an element of a cylindrical shell of Timoshenko type, interacting with a nonlinear elastic 
medium, in the class of stationary longitudinal-bending waves, leads to a nonlinear ordinary differential equation (8) for the normal displacement. The exact solitary-wave solutions in the form of a traveling pulse and traveling front are found for this equation. The numerical solutions found by means of finite difference method are in good agreement with the corresponding exact ones.

\section{Competing Interests}

The authors declare that they have no competing interests.

\section{Acknowledgments}

This research was supported by RFBR Grant 16-01-00176-a.

\section{References}

[1] M. Taj and J. Zhang, "Analysis of wave propagation in orthotropic microtubules embedded within elastic medium by Pasternak model," Journal of the Mechanical Behavior of Biomedical Materials, vol. 30, pp. 300-305, 2014.

[2] C. W. Lim and Y. Yang, "Wave propagation in carbon nanotubes: nonlocal elasticity-induced stiffness and velocity enhancement effects," Journal of Mechanics of Materials and Structures, vol. 5, no. 3, pp. 459-476, 2010.

[3] A. Muc, A. Banaş, and M. Chwal, "Free vibrations of carbon nanotubes with defects," Mechanics and Mechanical Engineering, vol. 17, no. 2, pp. 157-166, 2013.

[4] Q. Wang and V. K. Varadan, "Application of nonlocal elastic shell theory in wave propagation analysis of carbon nanotubes," Smart Materials and Structures, vol. 16, no. 1, pp. 178-190, 2007.

[5] L. H. Donell, Beams, Plates and Shells, McGraw-Hill, New York, NY, USA, 1976.

[6] J. D. Kaplunov, L. Y. Kossovich, and E. V. Nolde, Dynamics of Thin Walled Elastic Bodies, Academic Press, San Diego, Calif, USA, 1998.

[7] I. Andrianov and J. Awrejcewicz, Methods of Asymptotic Analysis and Synthesis in Nonlinear Dynamics and Mechanics of Solids, Institute of Computer Researches, Moscow, Russia, 2013.

[8] A. H. Nayfeh, Perturbation Methods, Wiley-Interscience, New York, NY, USA, 1973.

[9] Z. Qiu and H. Qiu, "A direct-variance-analysis method for generalized stochastic eigenvalue problem based on matrix perturbation theory," Science China Technological Sciences, vol. 57, no. 6, pp. 1238-1248, 2014.

[10] A. I. Zemlyanukhin and A. V. Bochkarev, "The perturbation method and exact solutions of nonlinear dynamics equations for media with microstructure," Computational Continuum Mechanics, vol. 9, pp. 182-191, 2016 (Russian).

[11] H.-S. Shen, "Thermal postbuckling analysis of imperfect Reissner-Mindlin plates on softening nonlinear elastic foundations," Journal of Engineering Mathematics, vol. 33, no. 3, pp. 259-270, 1998.

[12] M. Jabareen and I. Sheinman, "Dynamic buckling of a beam on a nonlinear elastic foundation under step loading," Journal of Mechanics of Materials and Structures, vol. 4, no. 7-8, pp. 13651373, 2009.

[13] V. I. Erofeev, V. V. Kazhaev, and N. P. Semerikova, Waves in Rods. Dispersion. Dissipation. Nonlinearity, Fizmatlit, Moscow, Russia, 2002 (Russian).
[14] G. A. Baker and P. R. Graves-Morris, Pade Approximants, Cambridge University Press, New York, NY, USA, 1996.

[15] S. P. Timoshenko and S. Woinowsky-Krieger, Theory of Plates and Shells, McGraw-Hill, New York, NY, USA, 1959.

[16] V. I. Erofeev, V. V. Kazhaev, E. E. Lisenkova, and N. P. Semerikova, "Nonsinusoidal bending waves in Timoshenko beam lying on nonlinear elastic foundation," Journal of Machinery Manufacture and Reliability, vol. 37, no. 3, pp. 230-235, 2008.

[17] R. Conte and M. Musette, The Painlevé Handbook, Springer, New York, NY, USA, 2008.

[18] R. J. LeVeque, Finite Difference Methods for Ordinary and Partial Differential Equations, Society for Industrial and Applied Mathematics (SIAM), Washington, DC, USA, 2007. 


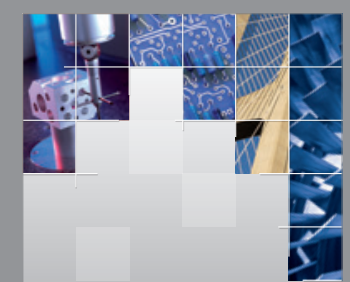

\section{Enfincering}
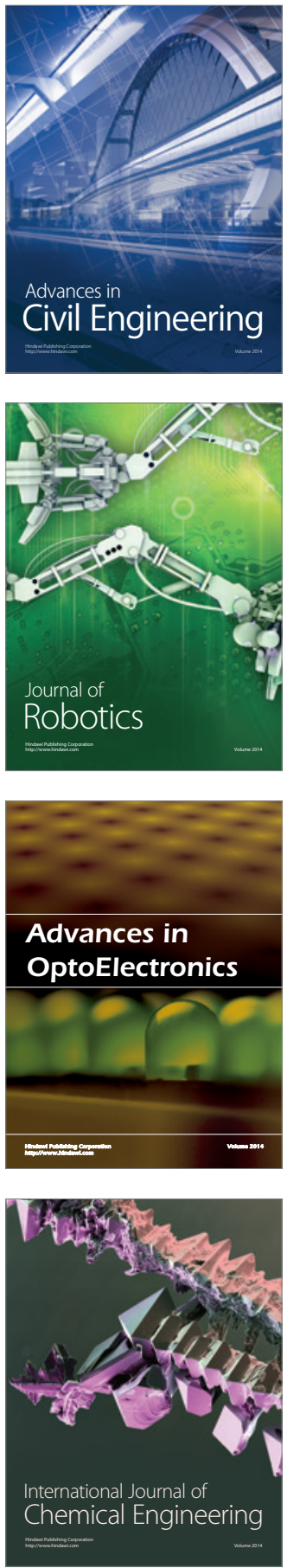

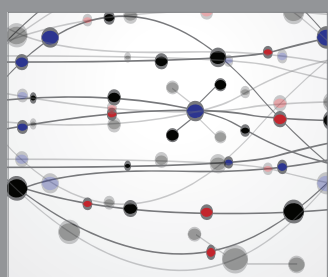

The Scientific World Journal

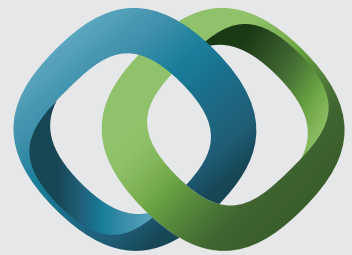

\section{Hindawi}

Submit your manuscripts at

http://www.hindawi.com
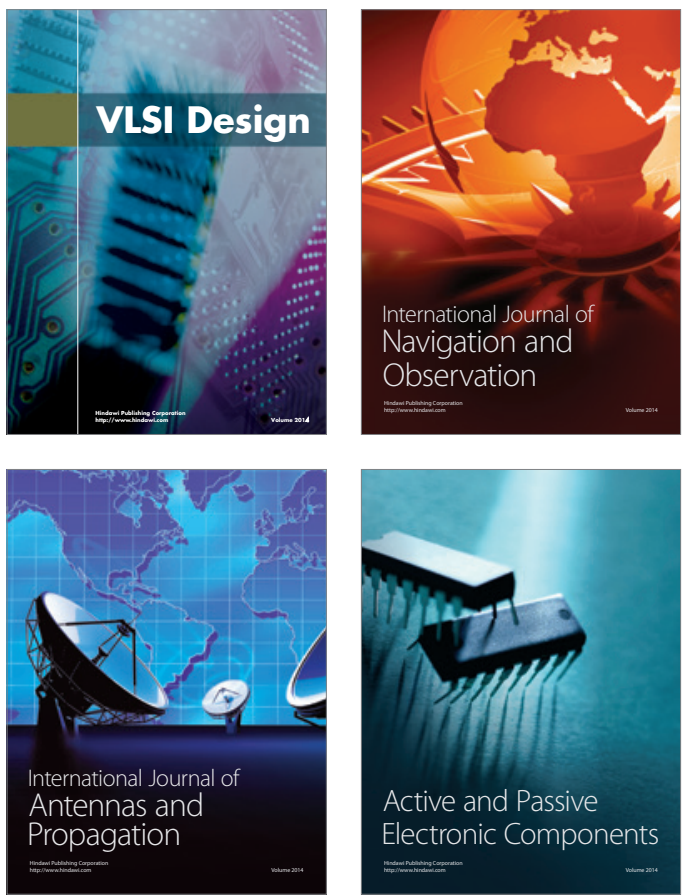
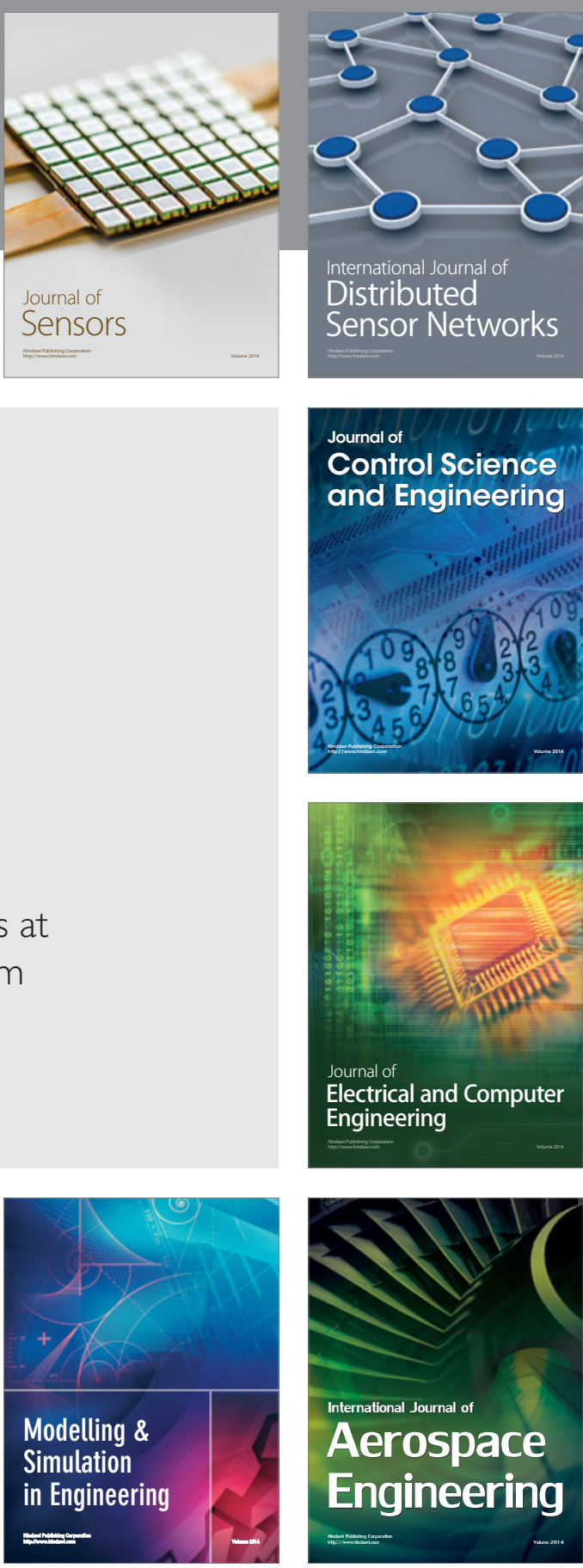

International Journal of

Distributed

Sensor Networks

Journal of

Control Science

and Engineering
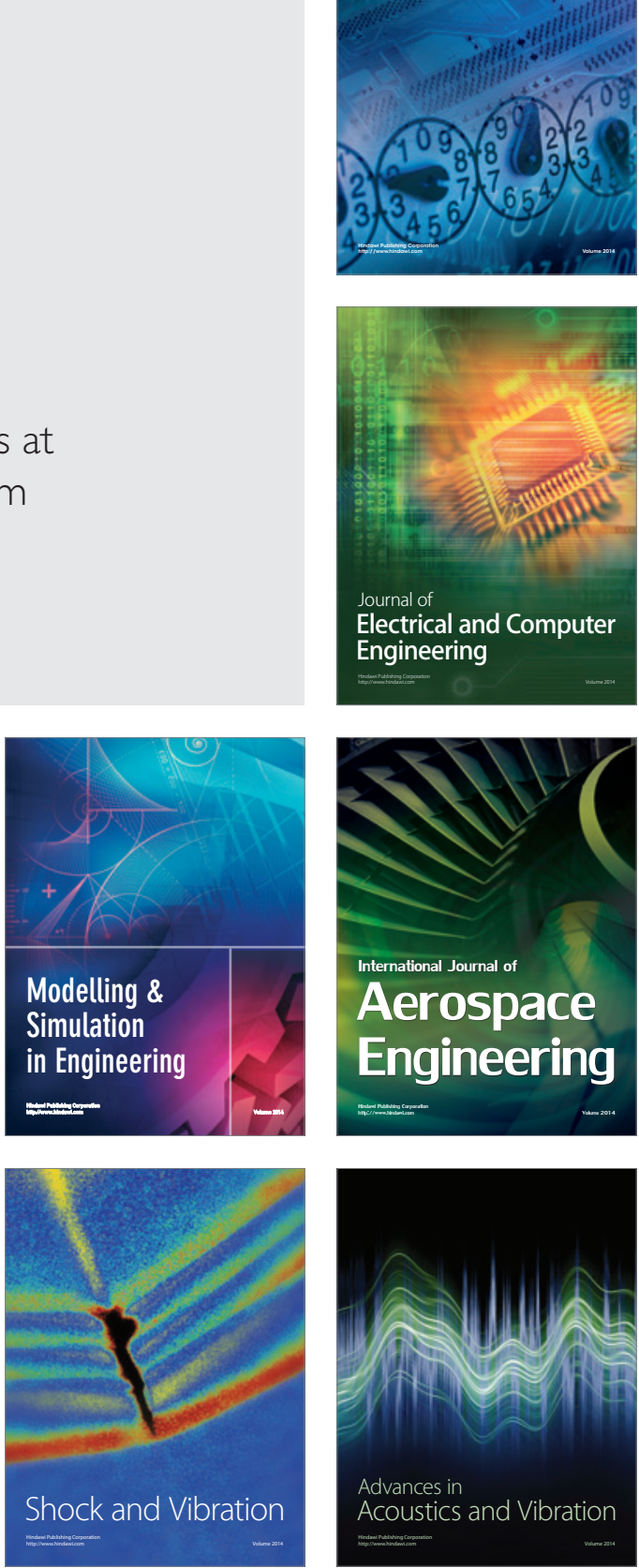